\title{
ANALYSE MICROSCOPIQUE DE L'ACTION DES SELS MERCURIQUES SUR LA CONCENTRATION PARASITAIRE EN COPROLOGIE
}

\author{
P. GENDRE*, J. BAILENGER**, B. DE FOUGIERES**
}

\begin{abstract}
RÉSUME. La fixation des ions mercuriques sur la couche utérine de la paroi des aufs d'Ascaris lumbricoides est démontrée, en microscopie optique, par une réaction histochimique et, en microscopie électronique, par microanalyse $\mathrm{X}$. Ces observations apportent des 'preuves à l'hypothèse d'une réaction possible entre les groupements de la surface des oufs et les constituants des réactifs de dilution, laquelle participe au mécanisme des méthodes de concentration en coprologie. Dans le cas des sels mercuriques, les groupements thiols libres, absents des différentes couches de la paroi des œufs d'Ascaris, ne constituent pas les éléments de la réaction.
\end{abstract}

Mots-clés : Ascaris. Concentrations. Coprologie.

\section{Microscopic analysis of mercuric salts action on parasitical concentration in copro- logy.}

SUMMARY. The fixation of mercuric ions on the uterin layer of the Ascaris lumbricoides egg wall is proved by light microscopic histochemical technics and electron microscopic microanalysis. These observations support the hypothesis of a possible reaction between the egg surface components and those of the dilution reagents. This reaction is used in the methods of concentration in coprology. In the case of mercuric salts, the free thiol components which are missing in all the Ascaris egg wall layers are not the factors of the reaction.

Key-words : Ascaris. Concentration. Coprology.

\section{Introduction}

La concentration des œufs d'helminthes, en coprologie, est réalisable par différentes méthodes parmi lesquelles sont décrites les techniques de flottation et celles que nous avons qualifiées de diphasiques (Bailenger, 1963). Nous avons précédemment montré (Bailenger, 1962, 1963, 1966, 1979), que leur principe ne

* Département de microscopie électronique, 351 , cours de la Libération, $F 33405$, Talence Cedex.
* Les demandes de tirage a part doivent être adressées au Laboratoire d'Organisation animale, parasitologie et mycologie humaines, Université Bordeaux II, place de la Victoire, F 33076 Bordeaux Cedex.

Accepté le 15 juillet 1986. 
repose pas seulement sur la densité relative des œufs par rapport à celle des réactifs de dilution, mais qu'intervient également la valeur de la balance hydrophilelipophile des éléments parasitaires : toute diminution de l'hydrophilie favorise la flottation, c'est-à-dire améliore la concentration ; inversement, une augmentation de l'hydrophilie facilite la sédimentation et accroît l'efficacité des procédés diphasiques. Nous avons émis l'hypothèse (Bailenger, 1966 ; Bailenger et coll., 1977) qu'il était possible de modifier la balance hydrophile-lipophile des œufs par une réaction entre leurs groupements de surface et les réactifs de dilution.

Nous nous proposons d'apporter des preuves supplémentaires à cette notion d'une réaction entre la surface des éléments parasitaires et les réactifs, en l'objectivant microscopiquement.

Comme élément parasitaire, nous choisissons les œufs d'Ascaris lumbricoides en raison des modifications importantes de leur comportement provoquées par les sels mercuriques (Bailenger et coll., 1977). Après les avoir mis au contact d'une solution de chlorure mercurique, nous recherchons le mercure par deux méthodes : 1) Coloration spécifique de $\mathrm{Hg}^{++}$et observation au microscope photonique; 2) Analyse au microscope électronique. Nous envisagerons ensuite la possibilité d'intervention des groupements thiols.

\section{Matériel et méthodes}

- Isolement et purification des ceufs D'Ascaris. La selle provenant d'un hôte parasité est délayée avec de l'eau distillée. La dilution est centrifugée à 3000 tours/minute pendant 5 minutes. Le culot est lavé à trois reprises avec de l'eau distillée, puis mis en suspension dans une solution saturée de chlorure de sodium ( $\left.d^{\mathrm{e}} 1,20\right)$. Après centrifugation à 2000 tours/minute, pendant 3 minutes, on aspire la partie liquide et on la dilue avec un égal volume d'une solution à $9 \%$ de chlorure de sodium. Une centrifugation de 3 minutes, à 2000 tours/ minute, concentre les oufs dans un culot ne renfermant que de très rares débris.

- Action D'un Sel mercurigue. On fait agir du chlorure mercurique, en solution aqueuse à $5 \%$, pendant 24 heures, soit sur des coupes à la congélation pour les observations en microscopie optique, soit sur des œufs entiers pour les études en microscopie électronique. Le matériel parasitaire traité est ensuite abondamment lavé à l'eau distillée.

- Coloration histochimique. Le mercure est recherché sur des coupes d'œufs épaisses de $5 \mu \mathrm{m}$ en appliquant la méthode de Brandino (6) : après action de quelques minutes d'une solution à $1 \%$ de diphénylcarbazide dans l'éthanol à $95 \%$

* Cette solution doit être conservée à l'abri de la lumière. 
et lavage à l'eau, la présence du mercure se traduit par une coloration violette.

Parallèlement, des coupes témoins, non soumises à l'action du mercure, subissent les mêmes traitements.

- Microscopie électronique. Le culot contenant les œufs est traité, pendant 3 heures, par de la glutaraldéhyde en solution à $3 \%$ dans un tampon phosphate 0,2 M. Après rinçage, on déshydrate par des bains d'alcool éthylique de titres croissants. On fait agir, ensuite, de l'oxyde de propylène puis on inclut à l'Epon. Les coupes ultrafines sont alors réalisées à l'ultratome LKB, puis recueillies sur des grilles de cuivre sans film support.

Ces coupes ultrafines sont, d'une part, étudiées en transmission et, d'autre part, soumises à une microanalyse $\mathrm{X}$.

Pour l'examen en transmission, les coupes sont contrastées par action de l'acétate d'uranyle et du citrate de plomb. On utilise un microscope électronique JEM 100 S pour l'observation.

Pour réaliser la microanalyse $X$, les coupes ne sont pas contrastées afin de réduire leur contamination. On les examine au microscope électronique JEOL 1200 Ex et le diagramme d'analyse est établi au moyen d'un microanalyseur TRACOR TN 2000.

On traite, parallèlement, des œufs soumis à l'action du mercure et des œufs témoins.

- Détection des groupements thiols libres. Kosower et coll. (1978, 1979, 1980), Gainer et Kosower (1980) ont décrit une nouvelle classe de substances de marquage, les bromobimanes, qui réagissent avec les groupes thiols pour donner des composés fortement fluorescents. Parmi ces dérivés, nous avons choisi le monobromobimane que nous utilisons sous forme du réactif suivant : le monobromobimane (P. M. 271,1) est dissous dans l'acétonitrile à la concentration de $50 \mathrm{mM}$ $(135,5 \mathrm{mg} / 10 \mathrm{ml})$. A partir de cette solution mère, d'une durée de conservation de 2 mois, on prépare extemporanément une dilution au $1 / 500^{\mathrm{e}}$ dans du tampon P. B. S. 7,35 (tampon phosphate $\mathrm{pH} 7,3510 \mathrm{mM}$; CINa $135 \mathrm{mM}$ ). Cette dilution, qui correspond à une solution $0,1 \mathrm{mM}$ de monobromobimane, doit être protégée de la lumière avant et pendant la réaction de marquage.

Les lames auxquelles adhèrent les sections d'œufs (traités ou non par le bichlorure de mercure) sont immergées pendant plusieurs minutes dans le tampon P. B. S. 7,35, à la température de la salle; on les recouvre ensuite avec la solution $0,1 \mathrm{mM}$ de monobromobimane et on laisse 10 à 20 minutes à l'obscurité, à la température de la salle. On lave avec du P. B. S. et l'on monte, entre lame et lamelle, avec du glycérol dilué au demi avec du tampon P. B. S.

L'examen a lieu avec un microscope à fluorescence muni d'un filtre d'excitation 355-425 nm (maximum d'absorption 370-385 nm) et d'un filtre d'émission de $460 \mathrm{~nm}$ (émission à 477-484 nm de couleur bleu turquoise). 


\section{Résultats}

La structure de la paroi des œufs d'Ascaris lumbricoides est relativement bien connue. Pour la décrire, nous adoptons la nomenclature de Bird (1971).

En microscopie électronique ( $P l .1,1$ et 2$)$, on observe notamment : - une couche externe, d'épaisseur variable (de quelques dixièmes de $\mu \mathrm{m}$ à quelques $\mu \mathrm{m}$ ) dénommée couche utérine; - une couche interne, d'épaisseur pratiquement constante aux environs de $0,3 \mu \mathrm{m}$, qui correspond à la couche chitineuse; à la jonction entre les deux, apparaissent deux lignes sombres séparées par un espace plus clair formant un ensemble de $0,05 \mu \mathrm{m}$ environ que l'on appelle la couche vitelline.

En microscopie optique ( $P l . I I, 1)$, la couche vitelline est trop mince pour être repérée. Par contre, on dissocie très nettement la couche utérine, brune et d'épaisseur variable, donnant aux œufs leur aspect mamelonné caractéristique, de la couche chitineuse incolore et régulière.

Après traitement par le mercure et coloration par la diphénylcarbazide $(P l . I I, 2)$, la couche utérine prend une teinte violette caractéristique du mercure qui s'oppose nettement à l'aspect incolore de la couche chitineuse.

En microscopie électronique, le traitement au mercure entraîne certains remaniements ultra-structuraux $(P l, I, 3$ et 4$)$ : les composants internes et externes de la couche vitelline sont moins osmiophiles; la couche chitineuse est épaissie et différencie une structure très riche en fibrilles, immédiatement au contact de la couche vitelline. Pour rechercher et localiser le mercure dans les couches pariétales des œufs traités, nous avons établi des diagrammes d'analyses X. Le diagramme global $(P l . I I I, 2)$ révèle la présence de mercure; l'examen des diagrammes de chacune des trois couches montre que ce métal se concentre exclusivement dans la couche utérine $(P l . I I I, 4)$, tandis qu'il n'est qu'à l'état de traces dans les couches sous-jacentes $(P l . ~ I I I, 5$ et 6$)$. Les autres pics qui apparaissent sur ces diagrammes correspondent à différents éléments : cuivre (dû à la grille du support); osmium (lié à la post-fixation); chlorure et calcium (éléments du milieu intestinal également présents dans les réactifs).

En raison de l'affinité de $\mathrm{Hg}^{++}$pour les groupements thiols, on peut envisager une réaction à leur niveau. Leur recherche, par la réaction de fluorescence au monobromobimane est négative dans les différentes couches pariétales (Pl. III, 3); la fluorescence bleu turquoise caractéristique des groupements thiols, qui s'observe sur la photographie, intéresse les constituants ovulaires adhérents à la paroi.

\section{Discussion}

La coloration histochimique du mercure et les diagrammes d'analyse $\mathbf{X}$ en microscopie électronique démontrent la fixation de $\mathrm{Hg}^{++}$au niveau de la paroi des œufs; cette fixation est strictement limitée à la couche utérine. Elle ne peut s'expliquer par une combinaison avec des groupements thiols puisqu'il ne semble 

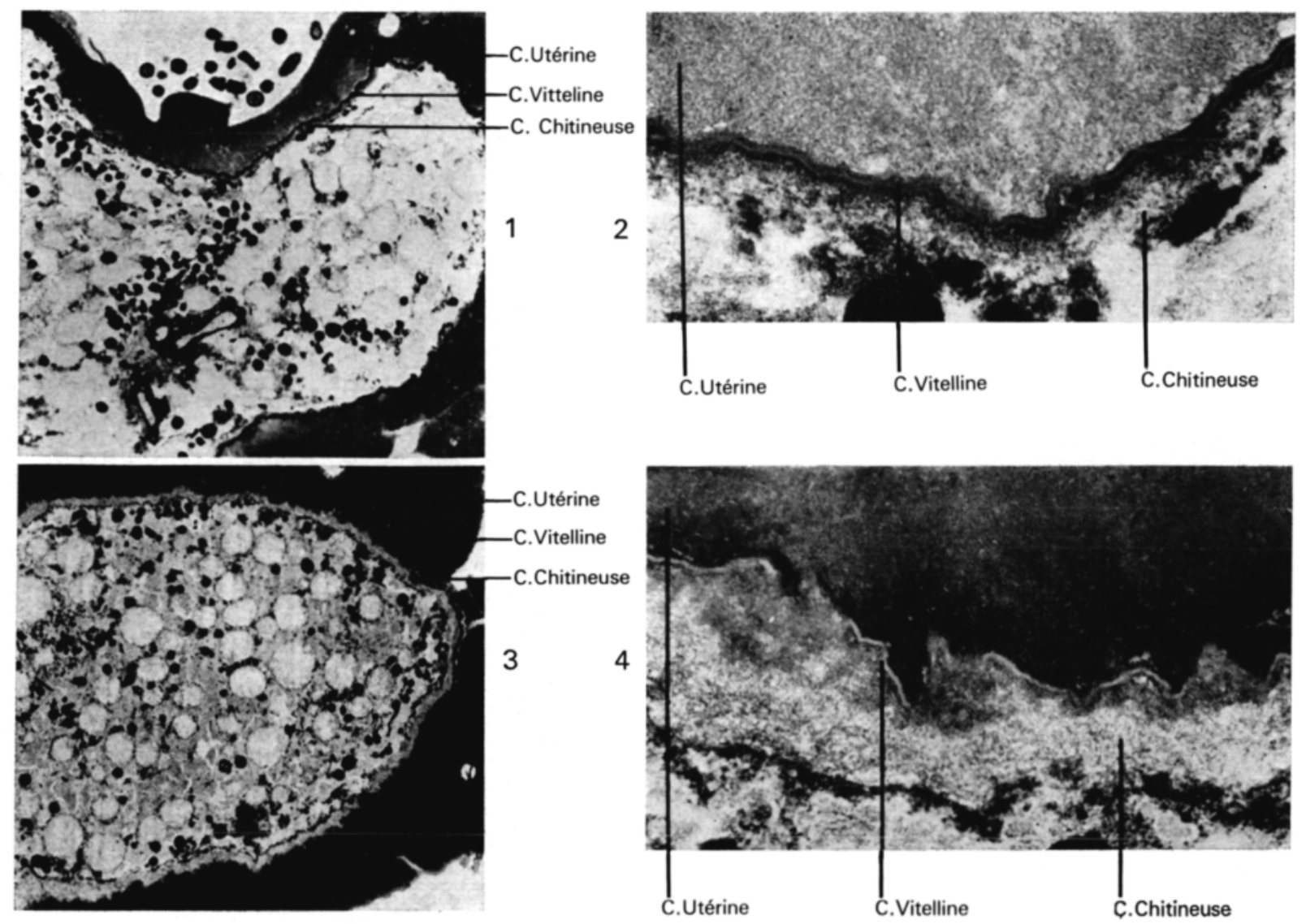

Pl. I. - Eufs d'Ascaris lumbricoides (microscopie électronique).

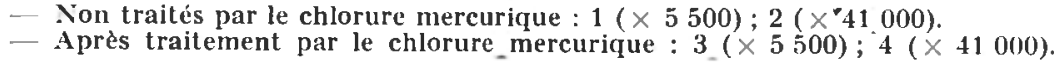



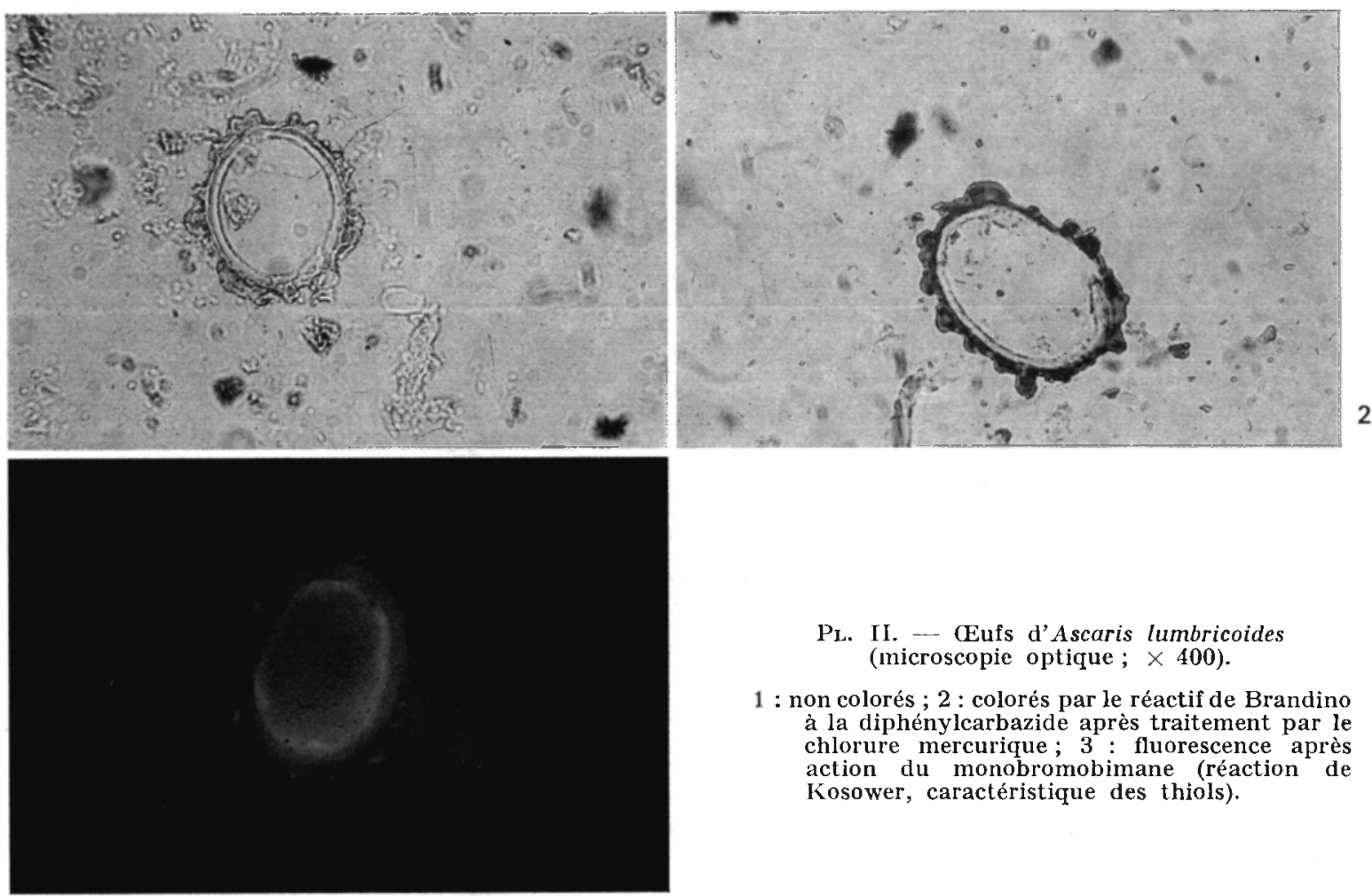

PL. II. - Eufs d'Ascaris lumbricoides (microscopie optique; $\times 400$ ).

1 : non colorés; 2 : colorés par le réactif de Brandino

à la diphénylcarbazide après traitement par le chlorure mercurique; 3 : fluorescence après action du monobromobimane (réaction de

Kosower, caractéristique des thiols). 
CARSOR: 8. 2000EV $=573$

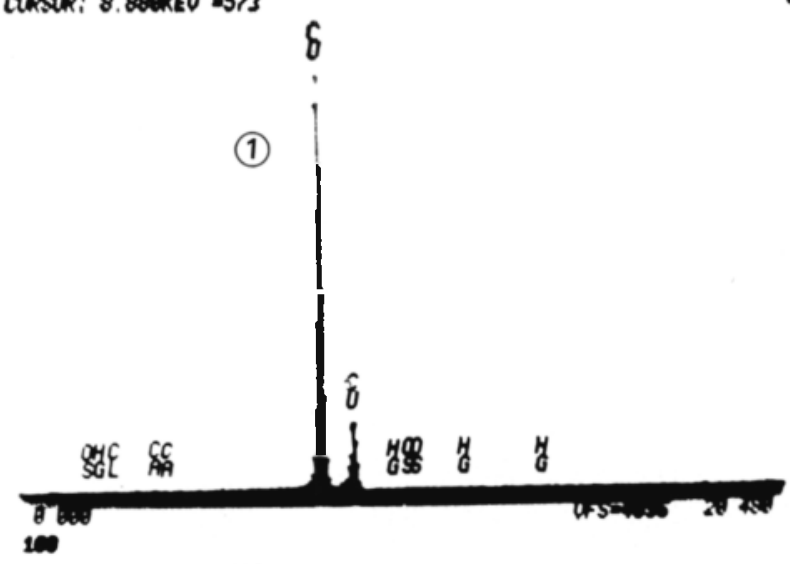

CURSOR: 4 MEV -389

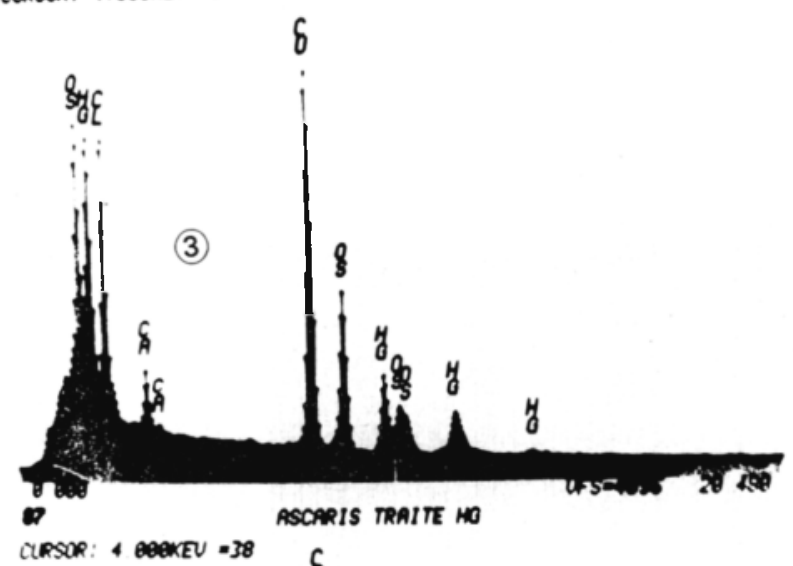

CIRSOR: 4 TEN $=38$
Cursor: 13.20012027

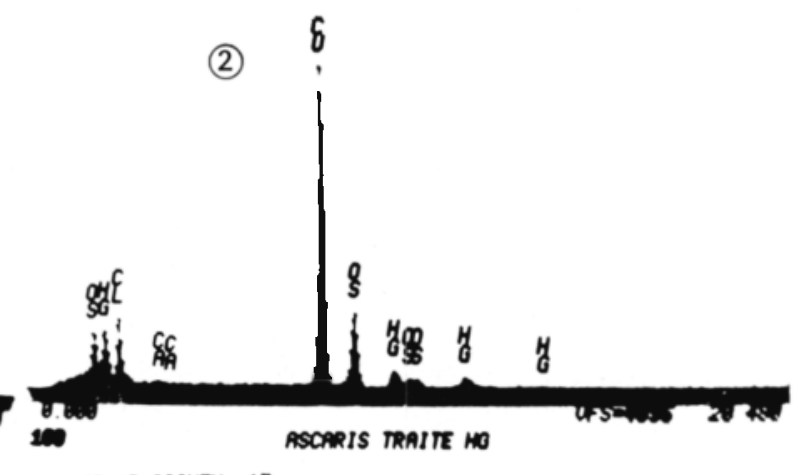

CURSOR: 13 ZOenEV $=17$

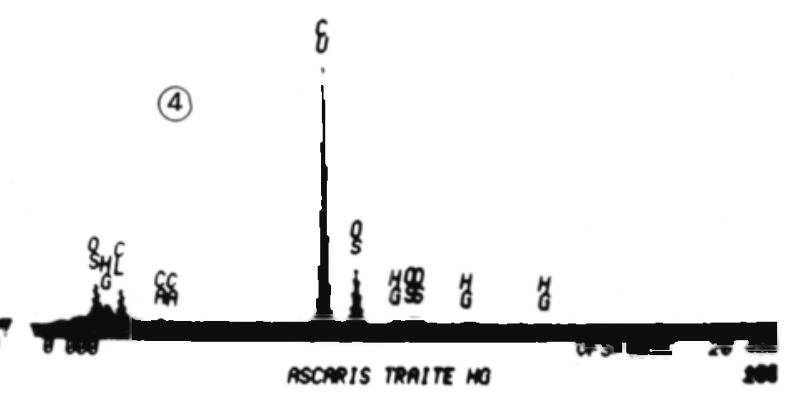

(5)

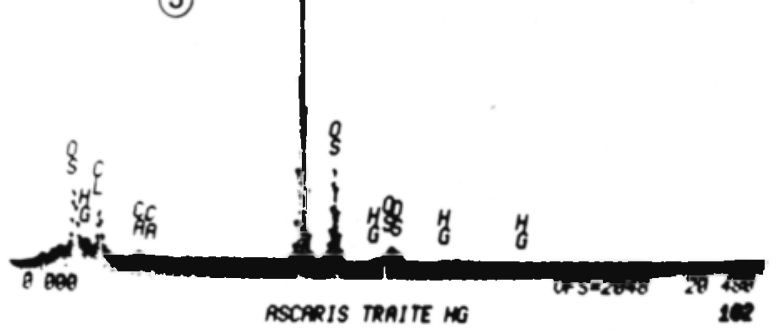

PL. III. - Diagrammes de microanalyse $\mathrm{X}$ (microscopie électronique) de la paroi des ceufs d'Ascaris lumbricoides. 1 : non traités. Après traitement au chlorure mercurique; 2 : analyse globale; 3 : couche utérine; 4 : couche vitelline ; 5 : couche chitineuse. 
pas exister de tels radicaux libres dans toute l'épaisseur de la paroi. Par contre, on peut envisager une réaction entre les ions mercuriques et certains acides aminés, notamment les diacides mono-aminés, tels l'acide glutamique, qui participent à la constitution de la couche utérine (Jaskoski, 1962).

Il existe donc bien des possibilités réactionnelles entre la paroi des cufs et les constituants des réactifs de dilution utilisés, en coprologie, dans les méthodes de concentration. De telles réactions participent aux mécanismes de la concentration en modifiant l'équilibre hydrophile-lipophile des éléments parasitaires. La nature des constituants d'un réactif de dilution est donc susceptible d'influencer l'efficacité d'une technique.

\section{BIBLIOGRAPHIE}

Bailenger J. : Valeur comparée des méthodes d'enrichissement en coprologie parasitaire humaine. Le Pharm. Biol., 1962, 3, 249-259.

Batlenger J. : Mécanisme et facteurs de concentration parasitaire dans les méthodes diphasiques en coprologie. Ann. Biol. Clin., 1963, 21, 805-816.

Bailenger J. : Les méthodes diphasiques de concentration parasitaire en coprologie. Ann. Parasitol. Hum. Comp., 1966, 4, 607-622.

BAIlenger J. : Mechanisins of parasitical concentration in coprology and their practical consequences. J. Am. Med. Technol., 1979, 41, 65-71.

Bailenger J., Puymartin G., Ca bannes A., Chanraud P. : Principe de la concentration parasitaire avec les méthodes par flottation et diphasiques; ses conséquences. Mécanisme d'action du mercure dans le réactif de Janeckso et Urbanyi. Le Pharm. Biol., 1977, 11, 267-277.

BIRD A. F. : The structure of Nematodes. Academic Press, Londres, 1971.

Brandino G. : Studi Sassari 1927, 5, 85. In: Histochimie animale (Lison L.). Gauthier-Villars, Paris, 1936.

Gaiver H., Kosower N. S. : Histochemical demonstration of Thiols and Disulfides by the fluorescent labelling agent, Monobromobimane: an application to the hypothalamo-neuro-hypophysial System. Histochemistry, 1980, 68, 309-315.

JAskoski B. J. : Paper chromatography of some fractions of Ascaris sumn eggs. Exp. Parasitol., $1962,12,19-24$.

Kosower N. S., Kosower E. M., Newton G. I., Ranney H. M. : Bimane fluorescent labels: labelling of normal human red cells under physiological conditions. Proc. Nat. Ac. Sc. U.S.A., $1979,76,3382-3386$.

Kosower N. S., Newton G. L., Kosower E. M., Ranney H. M. : Bimane fluorescent labels: characterization of the bimane labelling of human hemoglobin. Biochim. Biophys. Acta, $1980,622,201-209$.

Kosower E. M., Pazhenchevsky B., Hershkowitz E. : 1,5-Diazabicyclol (3,3,0) Octadienediones (9,10-Dioxabimanes). Strongly fluorescent syn-isomers. J. Am. Chem. Soc., 1978, $100,6516-6518$. 